\title{
Guidelines
}

Respiration 2014;87:513-521

Received: October 18, 2013

DOI: $10.1159 / 000360642$

Accepted after revision: February 14, 2014

Published online: April 24, 2014

\section{Expert Statement: Pneumothorax Associated with Endoscopic Valve Therapy for Emphysema - Potential Mechanisms, Treatment Algorithm, and Case Examples}

\author{
Arschang Valipour ${ }^{\mathrm{a}}$ Dirk-Jan Slebos ${ }^{\mathrm{b}}$ Hugo G. de Oliveira ${ }^{\mathrm{c}}$ Ralf Eberhardt ${ }^{\mathrm{d}}$ \\ Lutz Freitage Gerard J. Criner $^{f}$ Felix J.F. Herth ${ }^{d}$ \\ a Department of Respiratory and Critical Care Medicine, Ludwig Boltzmann Institute for COPD and Respiratory \\ Epidemiology, Otto Wagner Hospital, Vienna, Austria; ${ }^{b}$ Department of Pulmonary Diseases, University Medical \\ Center Groningen, University of Groningen, Groningen, The Netherlands; ' Serviço de Pneumologia do Hospital \\ de Clínicas de Porto Alegre, Universidade Federal do Rio Grande do Sul e Núcleo de Tratamento do Enfisema do \\ Hospital Moinhos de Vento, Porto Alegre, Brazil; ${ }^{d}$ Pneumology and Critical Care Medicine, Hospital Thoraxklinik, \\ University of Heidelberg, Translational Research Center Heidelberg, Heidelberg, and e Department of Interventional \\ Pneumology, West German Lung Center, University Hospital, University of Duisburg-Essen, Essen, Germany; \\ fDepartment of Medicine, Section of Pulmonary and Critical Care Medicine, Center for Inflammation, Translational \\ and Clinical Lung Research, Temple University, Philadelphia, Penn., USA
}

For editorial comment see p. 452

\section{Key Words}

Bronchoscopy - Emphysema - Bronchoscopic lung volume reduction Pneumothorax Endobronchial valves following these recommendations traumatic scenarios, prolonged drainage, extended hospitalizations, and/or surgery might be avoided in many cases.

두 2014 S. Karger AG, Basel

\begin{abstract}
The use of endoscopically placed unidirectional valves for the treatment of emphysema is increasing. With better patient selection, there is also an increased likelihood of complications associated with the procedure, such as postprocedural pneumothorax. There is, however, little evidence of pneumothorax management in patients with severe COPD and emphysema. This report describes an expert recommendation that has been developed to outline pneumothorax management after valve placement to inform physicians and patients of the risk-benefit profile and to assist them in decision making. Skilled and aggressive pneumothorax management is necessary in this patient population, and by
\end{abstract}

\section{Introduction}

Lung volume reduction surgery (LVRS) has been clearly shown to improve outcomes in specific patient subgroups of emphysema $[1,2]$. However, despite an estimated 3 million patients with emphysema in the USA, less than 15 LVRS operations are performed monthly [3]. Among the reasons for these numbers could be the unfavorable cost of the treatment and the reports of significant early mortality and morbidity associated with LVRS [2, 4]. Thus, few therapeutic options remain for this patient population and this unmet clinical need has spawned sev-

\section{KARGER}

E-Mail karger@karger.com www.karger.com/res (c) 2014 S. Karger AG, Basel

0025-7931/14/0876-0513\$39.50/0
Assoc. Prof. Arschang Valipour, MD, FCCP

Department of Respiratory and Critical Care Medicine

Ludwig Boltzmann Institute for COPD, Otto Wagner Hospital

Sanatoriumstrasse 2, AT-1140 Vienna (Austria)

E-Mail arschang.valipour@wienkav.at 
eral techniques to reduce the lung volume via minimally invasive endoscopic procedures. One such method is the insertion of unidirectional endobronchial valves (EBV) that aim to collapse hyperinflated lung regions by allowing trapped gas to escape from the targeted lobe while preventing gas refill during inhalation.

In the first multicenter, international, randomized, controlled trial with EBV (Pulmonx, Redwood City, Calif., USA) [5], modest improvements in the forced expiratory volume in $1 \mathrm{~s}\left(\mathrm{FEV}_{1}\right)$ and the 6 -min walk test were observed in patients treated with valves compared to controls. In a subgroup of patients, those with complete fissures on chest CT scans (a surrogate for the absence of interlobar collaterals) and optimal valve positioning, however, dramatically improved clinical outcomes were recorded. This group showed a $712-\mathrm{ml}$ reduction in target lobar volume, which was inversely correlated with the increase in $\mathrm{FEV}_{1}$. These observations were confirmed by the European cohort of the trial [6], where at 12 months subjects in the EBV group with complete fissure and complete lobar occlusion had a $28 \pm 32 \%$ improvement in $\mathrm{FEV}_{1}$ and a $22 \pm 40 \%$ improvement on the 6 -min walk test.

Like all interventional procedures, however, EBV therapy for emphysema is associated with a number of adverse events, such as postprocedural pneumothorax. This paper recommends an approach to pneumothorax management after valve placement to inform physicians and patients of the risk-benefit profile and to assist them in decision making. A more detailed evaluation of the staging and management of these patients is warranted and outlined below.

\section{Mechanism of Action of Valve Therapy-Related Pneumothorax in Emphysema}

Endobronchial one-way valves are designed to be placed unilaterally in the segments of a hyperinflated target lobe that has little or no collateral ventilation (fig. 1). The aim is to induce lobar volume reduction and improve the respiratory mechanics and thereby the underlying clinical symptoms [7]. Brown et al. [8] showed that a part of the volume reduction in the target lobe is redistributed to the ipsilateral lobe, with only a small portion redistributed to the contralateral lung. This result explains why the net reduction in total lung capacity in these patients is modest even with large reductions in the target lobe. In this patient population, the target lobe for volume reduction is usually the one with the most emphysematous destruction and the most severe hyperinflation. The occurrence

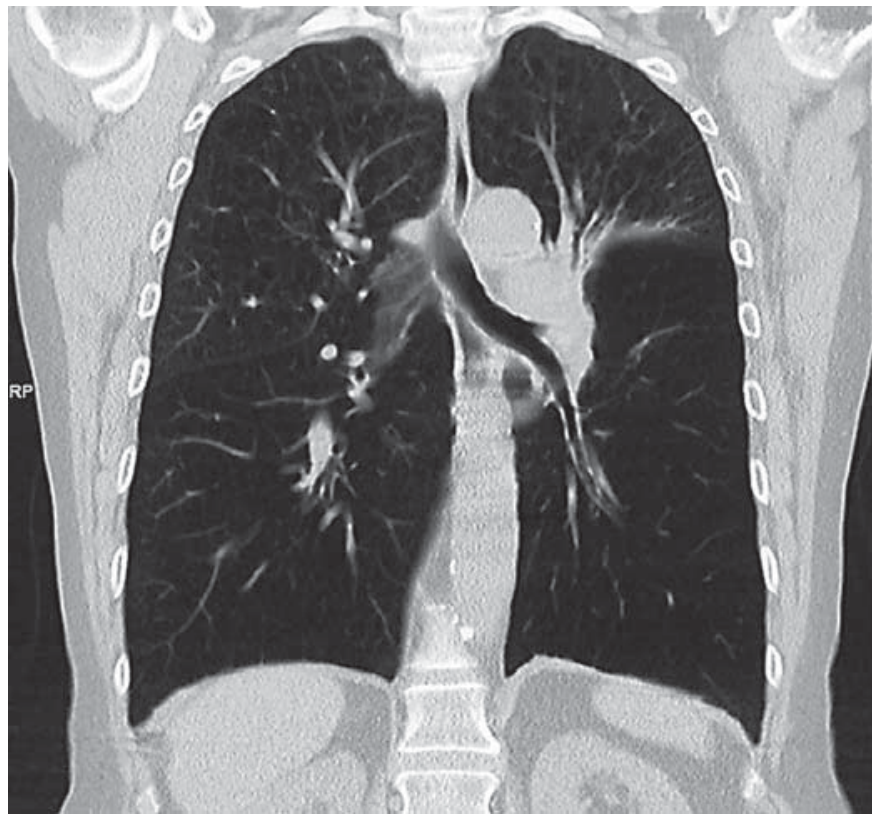

Fig. 1. Chest CT scan prior to endoscopic valve treatment of the hyperinflated left lower lobe.

of a pneumothorax following EBV therapy usually involves the untreated ipsilateral lobe and/or a collapsed target lobe that is unable to reexpand due to the presence of the one-way valves. It is hypothesized that postprocedurally the untreated ipsilateral lobe expands to occupy the newly created space in the thoracic cavity. The subsequent shifting of volumes on one side of the lung, which could be rapid in some cases, may be associated with tensioning and in some cases tearing of the already compromised lung tissue. The magnitude of the lobar volume shift, i.e. the proportional size of the target lobe reduction that is shifted to the nontreated ipsilateral lobe, might be a greater risk factor for pneumothorax; however, this hypothesis has not yet been formally tested. Rupture of blebs or bullae in the ipsilateral nontreated lobe is considered the most frequent cause of a pneumothorax [9] (fig. 2). Another mechanism could be parenchymal rupture as the lobes shift volumes due to preexisting pleural adhesion (fig. 3). All of the above conditions describe a pneumothorax that may result in a bronchopleural fistula that could cause pneumothorax expansion over time if not treated by chest tube insertion. Another less common but potential manifestation is pneumothorax ex vacuo. In this condition, acute lobar collapse results in a sudden increase in the negative intrapleural pressure surrounding the collapsed lobe (fig. 4). As a result, gas originating 


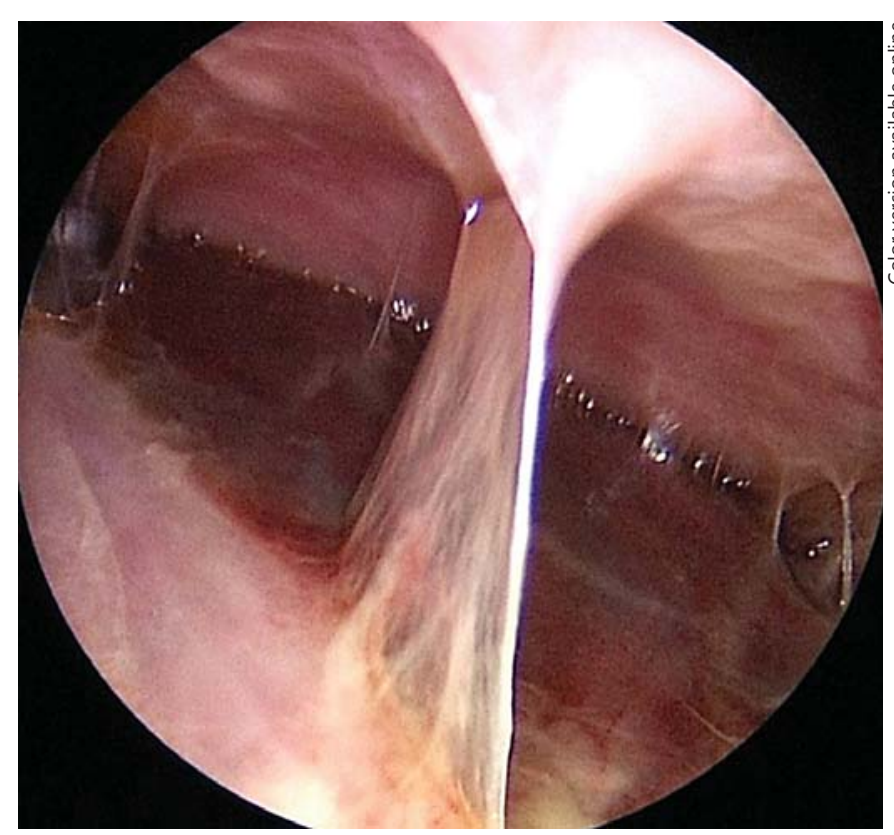

Fig. 2. Pleural adhesions as observed during video-assisted thoracoscopy.

from the ambient tissues and blood are drawn into the pleural space surrounding the collapsed lobe while the seal between the visceral and parietal pleura of the adjacent lobe or lobes remains intact [10]. In this latter condition, a bronchopleural fistula is not present and the pleural air would resolve spontaneously over time without the need for tube thoracostomy. Finally, a pneumothorax might theoretically also originate from the barotrauma response to the acute volume reduction in the treated lobe. The absence of a bronchopleural fistula might be due to the valves closing the originating bronchi. These cases of pneumothorax are expected to be associated with a lower volume on X-ray. A 'wait-and-see' policy might be successful in these patients.

\section{Pneumothorax Rate and Outcomes in Emphysema Patients Treated with Valves}

Pneumothorax is a common unintended consequence of numerous thoracic procedures. For example, the estimated risks of a pneumothorax during a CT-guided biopsy are highly variable; published reports range from $4 \%$ [11] to $42 \%$ [12]. In a large observational study [13] of patients undergoing transthoracic needle lung biopsy of a pulmonary nodule, the risk of any pneumothorax was

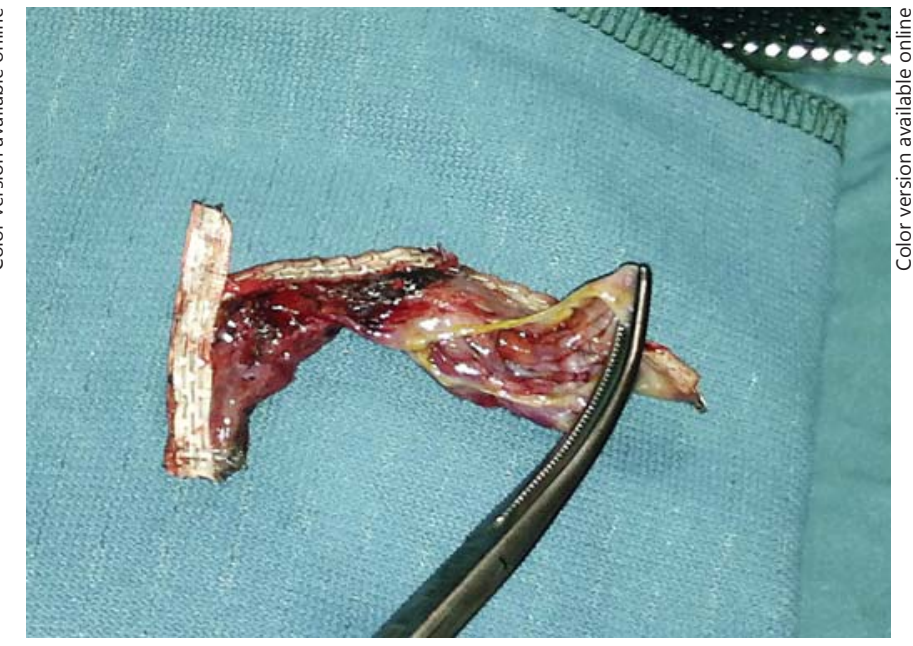

Fig. 3. Example of VATS-resected lung tissue with ruptured bullae after valve treatment that caused a persistent significant air leak.

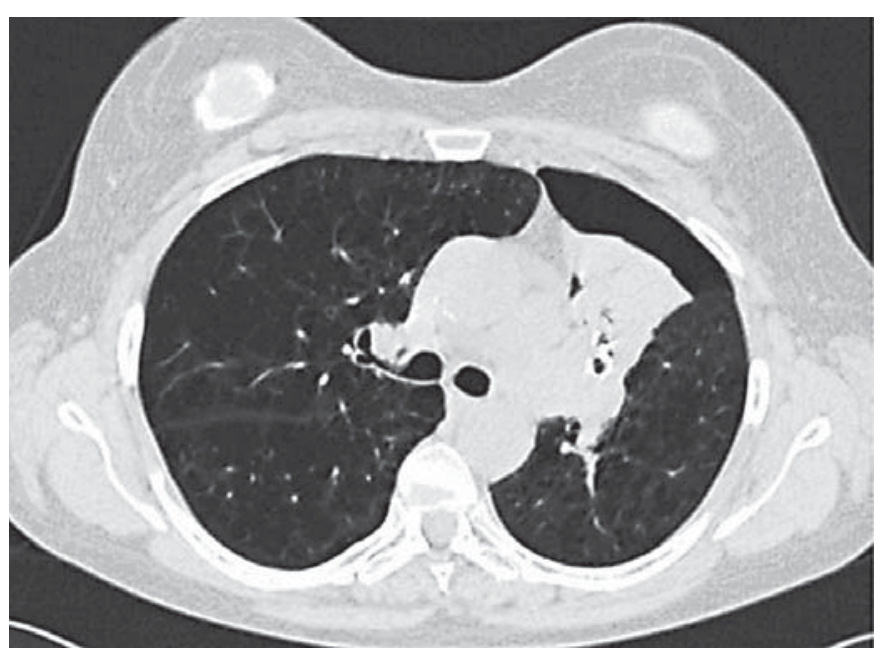

Fig. 4. Pneumothorax ex vacuo on a chest CT scan after valve treatment in the left upper lobe. The patient received no intervention. The pneumothorax was asymptomatic and resolved within 4 weeks.

$15 \%$, and $6.6 \%$ of all biopsies resulted in pneumothorax requiring a chest tube. Compared to patients without complications, those who experienced hemorrhage or pneumothorax requiring a chest tube had longer lengths of hospital stay and were more likely to develop respiratory failure requiring mechanical ventilation. Similarly, within 30 days of LVRS, $90 \%$ of patients developed air leaks [14]. Although the median duration of the air leaks was 7 days, $13 \%$ of the patients (66/496) had air leaks lasting 30 days or longer postoperatively [15]. 


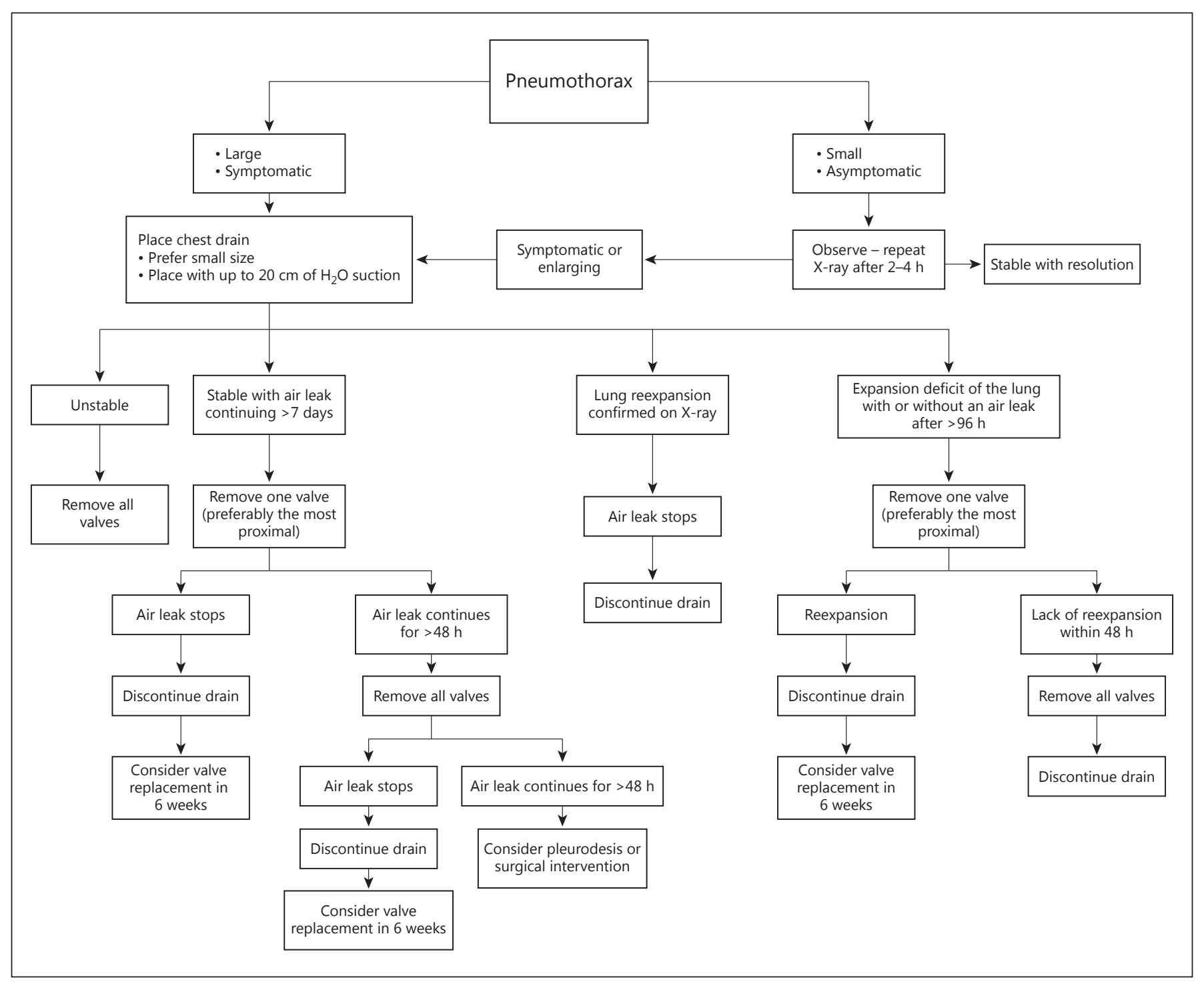

Fig. 5. Pneumothorax management recommendations. The recommended timelines in the proposed algorithm may differ individually depending on the patient's clinical status, the air leak volume, and/or the local resources and expertise.

The relationship between atelectasis and postinterventional pneumothorax was demonstrated in a case series of patients with emphysema using intrabronchial valves (Olympus, Tokyo, Japan) [16]. In that trial the overall incidence of pneumothorax was $12 \%$ during a 12 -month period. Analysis of those patients who underwent complete lobar exclusion (of the left upper lobe), however, revealed a pneumothorax rate of $29 \%$. Subsequently, studies using intrabronchial valves were designed to minimize the risk of pneumothorax with incomplete occlusion of the targeted upper lobes [17]. This approach resulted in higher safety, with no adverse events attributed to pneumothorax, but an overall lower efficacy and only a mild volume reduction of the treated lobe $(7 \pm 9 \%)$.

In the VENT trial, the pneumothorax rates within the first 90 days were unremarkable, i.e. $4.2 \%$ in the US cohort [5] and 4.5\% in the European cohort [6]. These low rates are not surprising given that $43.8 \%$ of the patients had incomplete lobar isolation due to suboptimal valve placement, and only $33 \%$ had complete fissures in the targeted lobes. Thus, only a small percentage of patients experienced significant target lobar volume reduction. The introduction of the Chartis pulmonary assessment system (Pulmonx) [18] enabled real-time assessment of the presence 
of collateral ventilation, and it has shown $75 \%$ accuracy in predicting which patients would respond to EBV therapy. Using this tool, there is an increased likelihood of response and the low pneumothorax rate from earlier reports is expected to rise. In fact, the authors of this report observe a pneumothorax rate of up to $30 \%$ in patients with lobar exclusion and no collateral ventilation in clinical practice.

In the current edition of Respiration, Gompelmann et al. [19], using controlled studies with EBV therapy both with and without use of the Chartis system, describe the occurrence of pneumothoraces that typically developed within the first 90 days of valve insertion. The median onset of a pneumothorax was 2 days after the procedure, with a median duration of 11.5 days. The majority of cases resolved either with observation or with the insertion of a chest tube. Importantly, these patients showed substantial reductions in the target lobar volume $(65 \pm 36 \%)$ at follow-up. This is of particular clinical importance as patients with a greater than $50 \%$ reduction in target lobar volume demonstrate clinically significant benefits in hyperinflation, exercise capacity, quality of life, and airflow obstruction [20]. The latter report further indicates results comparable to those of LVRS in these patients, with an expected lower morbidity and mortality. Therefore, in terms of patient outcomes, an approach with complete lobar occlusion and a higher risk of pneumothorax appears to be superior to incomplete lobar occlusion [21].

\section{Pneumothorax Management after Valve Treatment for Emphysema}

By definition, patients who are candidates for endoscopic valve therapy have severe emphysema with hyperinflation and impaired gas exchange and exercise capacity. Thus, these patients are less likely to tolerate a pneumothorax than patients with a primary spontaneous pneumothorax. Skilled and aggressive pneumothorax management is therefore warranted in this patient population and every pneumothorax, in particular a tension pneumothorax, can be life threatening [16]. The authors of this report introduce a management recommendation which is intended to provide guidance for physicians dealing with these cases in clinical practice (fig. 5). By following these recommendations, traumatic scenarios, prolonged drainage, extended hospital stays, and/or surgery might be avoidable in many cases.

Based on clinical signs, symptoms, and imaging results, the pneumothorax is classified as small or large (pneumothorax rim size between the lung and chest wall

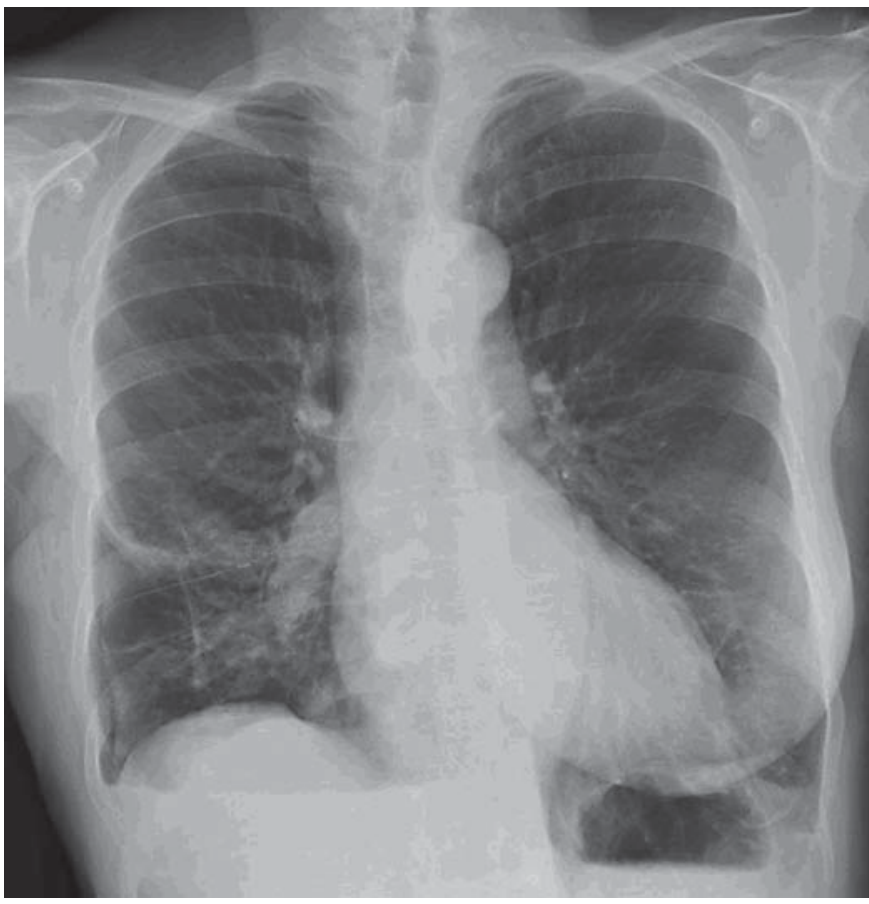

Fig. 6. Small pneumothorax after valve therapy of the right lower lobe. There was no existing or persistent bronchopleural fistula with evidence of volume loss on the right side. This patient was treated with observation alone.

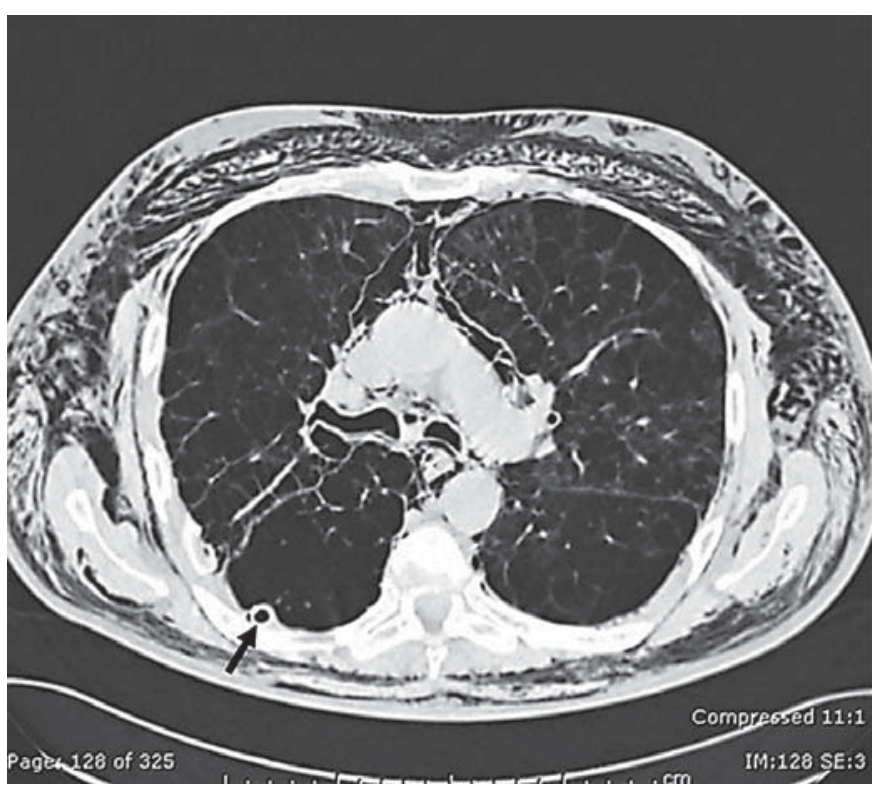

Fig. 7. Subcutaneous emphysema following pneumothorax after valve treatment in the right upper lobe. The patient received a large-bore chest tube (arrow head), valves were removed, the lung reexpanded, and the air leak disappeared after few days. This patient underwent repeated valve placement after 8 weeks. 


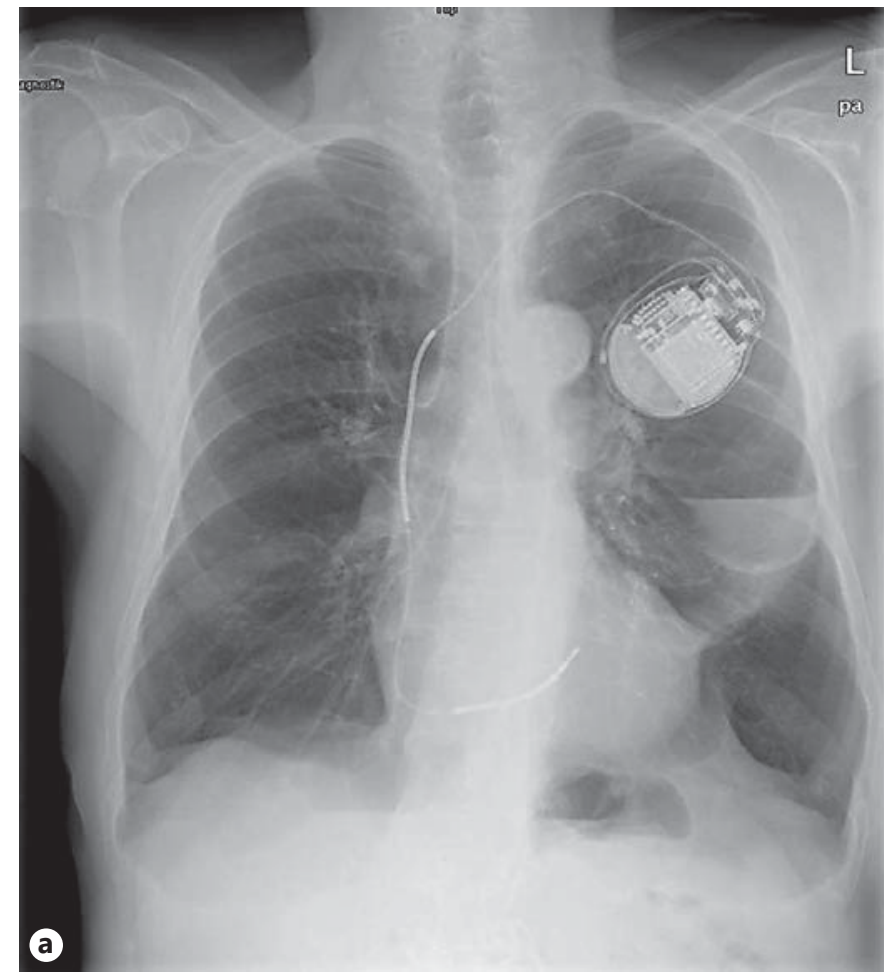

Fig. 8. a Interlobar pneumothorax after valve treatment in the left lower lobe. The patient developed clinical signs and symptoms of a pneumothorax $48 \mathrm{~h}$ after valve treatment of the left lower lobe. The X-ray shows an air-fluid level in the left mid-lung field. b Interlobar pneumothorax after valve treatment in the left lower lobe. A CT scan confirmed the interlobar pneumothorax. The patient

$<2 \mathrm{~cm}$ or $\geq 2 \mathrm{~cm}$, respectively) [22] and as symptomatic or asymptomatic:

- If the pneumothorax is small and asymptomatic, clinical observation may be sufficient and a repeat $\mathrm{X}$-ray is recommended within $4 \mathrm{~h}$ after the diagnosis and after 24 h (fig. 6).

- If clinical symptoms deteriorate or the size of the pneumothorax is enlarging, immediate insertion of a chest drain is required. Aspiration is less likely to be successful in patients with emphysema and severe airflow obstruction but can be considered in symptomatic patients with small pneumothoraces in an attempt to avoid chest drain insertion.

- The insertion of a small-bore chest drain is recommended by the British Thoracic Society [22]; however, patients may develop a tension pneumothorax, respiratory failure, and/or massive soft tissue emphysema and a large-bore chest tube may be necessary in these cases (fig. 7).

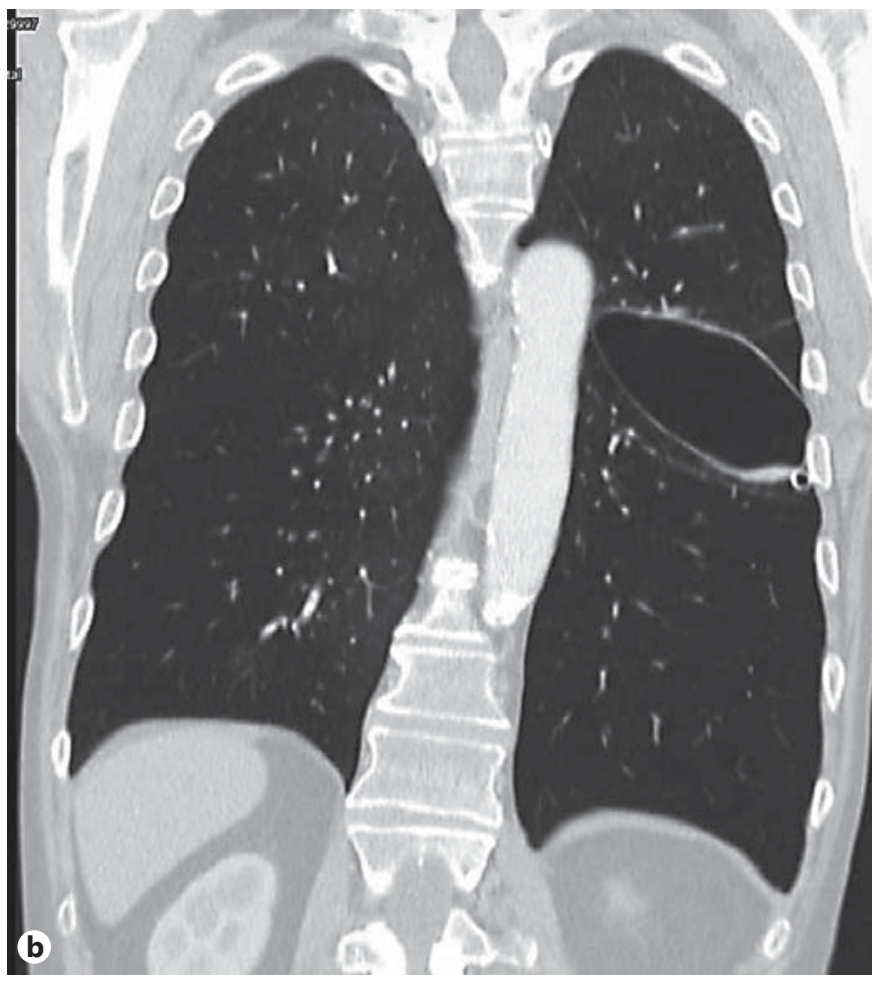

received a chest tube in the pleural cavity prior to the CT scan, which did not result in resolution of the pneumothorax. The patient, however, was discharged with the interlobar pneumothorax without any further symptoms after removal of the chest tube. Notably, there was no evidence of volume reduction after pneumothorax occurrence.
- While the diagnosis is usually confirmed on a chest radiograph, a chest CT scan may be necessary in order to confirm the localization (e.g. interlobar pneumothorax, see fig. 8a, b) and/or extent (e.g. complete or partial) of the pneumothorax. Furthermore, a CT scan is recommended to guide treatment options and/or rule out aberrant chest tube placement.

- General measures: oxygen is indicated but caution is required for patients with carbon dioxide retention [22]. In addition, physicians should make sure that patients receive appropriate bronchodilator therapy and consider systemic steroid and antibiotic therapy (a treatment course of 5-7 days is recommended).

As the pneumothorax usually occurs in the untreated ipsilateral lobe, the decision of whether or not to remove one or all of the inserted valves must be made, as the chest drain and suction cannot inflate the treated lobe due to the presence of the valves. This decision must be made taking the clinical symptoms, the amount of air leak vol- 
ume, and the existence or absence of soft tissue emphysema into account:

- Removing one or more valves might return the target lobe to its (hyperinflated) pretreatment state and promote pneumothorax healing by reestablishing pleural contact. The lack of full expansion and pleural sealing of the untreated lobe might, however, also be due to endobronchial mucus plugging requiring bronchoscopic suction.

- If the air leak stops after valve removal and does not reappear after discontinuation of the chest drain, valve replacement can be considered 6-8 weeks after chest tube removal. This recommendation is based on the data presented by Gompelman et al. [19], in which patients with a pneumothorax experienced substantial functional improvements. However, despite evidence of atelectasis and subsequent pneumothorax after valve treatment, the initial volume reduction may disappear after pneumothorax resolution. In these cases, repeat bronchoscopy to check for proper valve placement may be recommendable. If the valves are in proper placement, no further action is required and the patient needs to be informed about the potential risk of a repeat pneumothorax.

- The likelihood of a repeat pneumothorax appears to be very low in clinical practice, most likely due to pleural adhesion after the first occurrence; however, there is no scientific data to support this hypothesis.

\section{Prolonged Air Leakage after Pneumothorax}

In cases with a persistent air leak for at least 1 week despite valve removal, the following treatment options for a prolonged air leak need to be considered: mechanical or chemical pleurodesis, use of a Heimlich valve, or surgery. The timing and choice of the therapeutic approach may largely depend on the patient's condition and the experience with and availability of these treatment options at the respective institution. In patients with severe heterogeneous emphysema, the surgical approach might be conducted with the intent of volume reduction, though high rates of cardiopulmonary morbidity and mortality need to be taken into consideration [23].

The majority of patients with a valve-associated pneumothorax, however, do not require surgical interventions and can be safely treated with a chest tube with or without a Heimlich valve in the presence of a prolonged air leak.

Notably, recent case series have demonstrated the efficacy of valve insertion in reducing and eliminating air leaks $[24,25]$. Thus, valve insertion in targeted airways leading to the region of parenchymal air leakage might be a potential alternative treatment approach if isolation of the 'responsible' airways is feasible.

\section{Postprocedural Detection of Pneumothorax}

A chest X-ray examination is strongly recommended within the first $4 \mathrm{~h}$ and then $24 \mathrm{~h}$ after the procedure as routine clinical practice. As the majority of pneumothoraces following valve placement occur within $48 \mathrm{~h}$ [19], the authors of this report recommend that patients stay in hospital for at least 2 nights after the procedure for surveillance. It has to be acknowledged, however, that institutional practices may substantially vary and patients might be discharged earlier if stable after the procedure. Thus, importantly, physicians should inform their patients of the potential risk of a pneumothorax after discharge, the symptoms to be aware of, and the information to provide to emergency responders. Patients requiring air travel immediately after discharge might require a longer period of postprocedural observation (up to 5 days).

A late occurrence of pneumothorax, i.e. after discharge, could be especially serious depending on the patient's circumstances and the clinical development of the pneumothorax. Since the valves cause gradual shrinkage of the treated lobe and thereafter only maintain the collapse, they may not increase the risk of pneumothorax when the process of lobar volume shift has been completed. Hence, a pneumothorax occurring 3 months after endoscopic valve therapy is less likely to be due to valve placement. There is limited experience in this category; however, any pneumothorax in patients with emphysema and limited respiratory reserves needs to be considered an emergency and patients should be informed of the need to seek medical help when presenting with any signs or symptoms of a pneumothorax regardless of the timing of valve therapy. Ideally, written information sheets with details of the signs and symptoms of a pneumothorax and guidance on how and when to seek medical help should be provided.

\section{Limitations of This Recommendation}

This consensus statement has a number of limitations. The data related to pneumothorax occurrence after valve treatment is based on retrospective analyses from recently published reports. The recommendations 
presented here, however, are largely derived from clinical experience and a consensus of expert opinion rather than scientific evidence or intervention studies. Thus, further research into both baseline predictors of a pneumothorax and management strategies should be performed, which might help to optimize the risk-benefit ratio of patients undergoing valve therapy for lung volume reduction.

\section{Conclusion}

The occurrence of a pneumothorax and its management should be considered routine clinical sequelae of bronchoscopic lung volume reduction in patients who have progressive disease, compromised lung tissue, and limited therapeutic options. With this in mind, the authors would like to propose a rational management plan that attempts to guide physicians in daily practice. Given optimized patient selection, the risk-benefit ratio of a pneumothorax appears to be acceptable, as the majority of these patients develop substantial improvements in functional outcomes after resolution of the pneumothorax.

\section{Acknowledgements}

The authors of this report are indebted to the Department of Radiology at the Otto Wagner Hospital in Vienna (Prof. G.H. Mostbeck), at the Thoraxklinik Heidelberg (Prof. C.P. Heussel), and at the University Medical Center Groningen for the use of chest X-ray and CT scan images to illustrate this expert recommendation. The authors would further like to acknowledge Ms. Caroline Van der Wauwer, Department of Thoracic Surgery, University Medical Center Groningen, for providing the VATS images.

We would like to acknowledge Sri Radhakrishnan from Pulmonx International for his technical writing support.

\section{Financial Disclosure and Conflicts of Interest}

L.F. has ownership interest in Pulmonx. F.J.F.H. has been in advisory board meetings of Pulmonx. R.E. received fees for educational activities from Pulmonx and Olympus. A.V. has served as a consultant for Pulmonx and received lecture fees from both Pulmonx and Olympus Europe. D.-J.S. has been in advisory board meetings and received lectures fees and travel support from Pulmonx for presentation of scientific data.

\section{References}

1 Geddes D, Davies M, Koyama H, Hansell D, Pastorino U, Pepper J, Agent P, Cullinan P, MacNeill SJ, Goldstraw P: Effect of lung-volume-reduction surgery in patients with severe emphysema. N Engl J Med 2000;343:239-245.

- 2 Fishman A, Martinez F, Naunheim K, et al; National Emphysema Treatment Trial Research Group: A randomized trial comparing lung-volume-reduction surgery with medical therapy for severe emphysema. N Engl J Med 2003;348:2059-2073.

-3 Berger RL, DeCamp MM, Criner GJ, Celli BR: Lung volume reduction therapies for advanced emphysema: an update. Chest 2010; 138:407-417.

-4 Naunheim KS, Wood DE, Krasna MJ, DeCamp MM Jr, Ginsburg ME, McKenna RJ Jr, Criner GJ, Hoffman EA, Sternberg AL, Deschamps C; National Emphysema Treatment Trial Research Group: Predictors of operative mortality and cardiopulmonary morbidity in the National Emphysema Treatment Trial. J Thorac Cardiovasc Surg 2006;131:43-53.

$\checkmark 5$ Sciurba FC, Ernst A, Herth FJ, Strange C, Criner GJ, Marquette CH, Kovitz KL, Chiacchierini RP, Goldin J, McLennan G; VENT Study Research Group: A randomized study of en- dobronchial valves for advanced emphysema. N Engl J Med 2010;363:1233-1244.

-6 Herth FJ, Noppen M, Valipour A, Leroy S, Vergnon JM, Ficker JH, Egan JJ, Gasparini S, Agusti C, Holmes-Higgin D, Ernst A; International VENT Study Group: Efficacy predictors of lung volume reduction with Zephyr valves in a European cohort. Eur Respir J 2012;39:1334-1342.

7 Herth FJF, Gompelmann D, Ernst A, Eberhardt R: Endoscopic lung volume reduction. Respiration 2009;79:5-13.

-8 Brown MS, Kim HJ, Abtin FG, Strange C, Galperin-Aizenberg M, Pais R, Da Costa IG, Ordookhani A, Chong D, Ni C, McNitt-Gray MF, Tashkin DP, Goldin JG: Emphysema lung lobe volume reduction: effects on the ipsilateral and contralateral lobes. Eur Radiol 2012;22:1547-1555.

-9 Shen KR, Cerfolio RJ: Decision making in the management of secondary spontaneous pneumothorax in patients with severe emphysema. Thorac Surg Clin 2009;19:233-238.

10 Woodring JH, Baker MD, Stark P: Pneumothorax ex vacuo. Chest 1996;110:1102-1105.

-11 Laspas F, Roussakis A, Efthimiadou R, Papaioannou D, Papadopoulos S, Andreou J: Per- cutaneous CT-guided fine-needle aspiration of pulmonary lesions: results and complications in 409 patients. J Med Imaging Radiat Oncol 2008;52:458-462.

12 Hiraki T, Mimura H, Gobara H, Shibamoto K, Inoue $\mathrm{D}$, Matsui $\mathrm{Y}$, et al: Incidence of and risk factors for pneumothorax and chest tube placement after CT fluoroscopy-guided percutaneous lung biopsy: retrospective analysis of the procedures conducted over a 9-year period. AJR Am J Roentgenol 2010;194:809-814.

13 Wiener RS, Schwartz LM, Woloshin S, Welch HG: Population-based risk for complications after transthoracic needle lung biopsy of a pulmonary nodule: an analysis of discharge records. Ann Intern Med 2011;155:137-144.

14 DeCamp MM, Blackstone EH, Naunheim KS, Krasna MJ, Wood DE, Meli YM, McKenna RJ: Patient and surgical factors influencing air leak after lung volume reduction surgery: lessons learned from the National Emphysema Treatment Trial. Ann Thorac Surg 2006;82: 197-207.

15 Criner GJ, Sternberg Al: National Emphysema Treatment Trial: the major outcomes of lung volume reduction surgery in severe emphysema. Proc Am Thorac Soc 2008;5:393. 
16 Sterman DH, Mehta AC, Wood DE, Mathur PN, McKenna RJ Jr, Ost DE, Truwit JD, Diaz P, Wahidi MM, Cerfolio R, Maxfield R, Musani AI, Gildea T, Sheski F, Machuzak M, Haas AR, Gonzalez HX, Springmeyer SC; IBV Valve US Pilot Trial Research Team: A multicenter pilot study of a bronchial valve for the treatment of severe emphysema. Respiration 2010;79:222-233.

17 Ninane V, Geltner C, Bezzi M, Foccoli P, Gottlieb J, Welte T, Seijo L, Zulueta JJ, Munavvar M, Rosell A, Lopez M, Jones PW, Coxson HO, Springmeyer SC, Gonzalez X: Multicentre European study for the treatment of advanced emphysema with bronchial valves. Eur Respir J 2012;39:1319-1325.

18 Herth FJF, Eberhardt R, Gompelmann D, Ficker JH, Wagner M, Ek L, Schmidt B, Slebos DJ: Radiological and clinical outcomes of using Chartis ${ }^{\mathrm{TM}}$ to plan endobronchial valve treatment. Eur Respir J 2013;41:302-308.
19 Gompelmann D, Herth FJF, Slebos DJ, Valipour A, Ernst A, Criner GJ, Eberhardt R: Pneumothorax following endobronchial valve therapy and impact on clinical outcomes in severe emphysema. Respiration 2014;87:485-491.

20 Valipour A, Herth FJF, Burghuber OC, Criner G, Vergnon J-M, Goldin J, Sciurba F, Ernst A; VENT study group: Target lobe volume reduction and COPD outcome measures after endobronchial valve therapy. Eur Respir J 2014;43:387-396.

21 Eberhardt R, Gompelmann D, Schuhmann M, Heussel CP, Herth FJ: Complete unilateral vs partial bilateral endoscopic lung volume reduction in patients with bilateral lung emphysema. Chest 2012;142:900-908.

22 MacDuff A, Arnold A, Harvey J; BTS Pleural Disease Guideline Group: Management of spontaneous pneumothorax: British Thoracic Society Pleural Disease Guideline 2010. Thorax 2010;65(suppl 2):ii18-ii31.
23 Naunheim KS, Wood DE, Krasna MJ, DeCamp MM Jr, Ginsburg ME, McKenna RJ Jr, Criner GJ, Hoffman EA, Sternberg AL, Deschamps C; National Emphysema Treatment Trial Research Group: Predictors of operative mortality and cardiopulmonary morbidity in the National Emphysema Treatment Trial. J Thorac Cardiovasc Surg 2006;131:43-53.

24 Travaline JM, McKenna RJ, De Giacomo T, Venuta F, Hazelrigg SR, Boomer M, Criner GJ: Treatment of persistent pulmonary air leaks using endobronchial valves. Chest 2009; 136:355-360.

25 Firlinger I, Stubenberger E, Müller MR, Burghuber OC, Valipour A: Endoscopic one-way valve implantation in patients with prolonged air leak and the use of digital air leak monitoring. Ann Thorac Surg 2013;95:1243-1249. 